\title{
Electric and Magnetic Properties Computed for Valence Bond Structures: Is There a Link between Pauling Resonance Energy and Ring Current?
}

\author{
Remco W. A. Havenith \\ Debye Institute, Theoretical Chemistry Group, ${ }^{\dagger}$ Utrecht University, Padualaan 8, \\ 3584 CH Utrecht, The Netherlands \\ r.w.a.havenith@chem.uu.nl
}

Received February 7, 2006

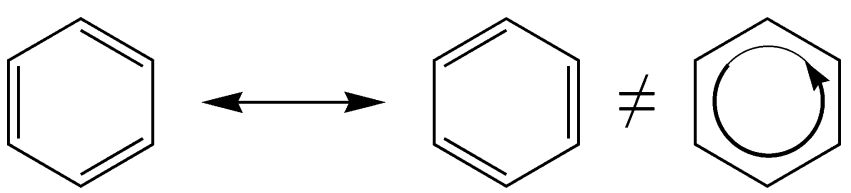

To establish the link between the aromaticity descriptors based on the Pauling resonance energy and the molecular properties, the electric (polarizability) and magnetic (magnetizability) field response properties have been calculated using the valence bond approach for various molecules and their individual Kekulé resonance structures. The results show that there is no direct relationship between the Pauling resonance energy and the properties; the response properties are weighted averages of the properties of the individual structures. According to the aromaticity criteria based on molecular properties, one-structure benzene would be aromatic; thus, concerning molecular properties, spin-coupled bonds do not behave like localized bonds in Lewis structures, with which they are usually associated.

\section{Introduction}

In the ongoing debate on aromaticity and how it should be defined, ${ }^{1-5}$ the various descriptors based on molecular properties, in particular, magnetic (induced current density, ${ }^{6-8}$ exalted magnetizability, ${ }^{4} \mathrm{NMR}$ chemical shifts, nucleus-independent chemical shielding (NICS) ${ }^{9}$ ) and electric properties (polarizabilities), ${ }^{10}$ are continuously compared to aromatic stabilization energies (ASE) ${ }^{11}$ and resonance energies. All criteria try to establish the resemblance of a compound with benzene. The resonance criterion is based on the picture of two 1,3,5-

$\dagger$ Affiliated to Organic Chemistry and Catalysis.

(1) Special issue on aromaticity: Chem. Rev. 2001, 101, 1115-1566. 343 .

(2) Special issue on aromaticity: Phys. Chem. Chem. Phys. 2004, 6, 217-

(3) Cyrañski, M. K.; Krygowski, T. M.; Katritzky, A. R.; von R. Schleyer, P. J. Org. Chem. 2002, 67, 1333-1338.

(4) von R. Schleyer, P.; Jiao, H. Pure Appl. Chem. 1996, 68, 209-218.

(5) Special issue on delocalization and conjugation: Chem. Rev. 2005, $105,3433-3947$.

(6) London, F. J. Phys. Radium 1937, 8, 397-409.

(7) Pauling, L. J. Chem. Phys. 1936, 4, 673-677.

(8) Pople, J. J. Chem. Phys. 1956, 24, 1111.

(9) von R. Schleyer, P.; Maerker, C.; Dransfeld, A.; Jiao, H.; van Eikema Hommes, N. J. R. J. Am. Chem. Soc. 1996, 118, 6317-6318.

(10) Lazzeretti, P.; Tossell, J. A. J. Mol. Struct. (THEOCHEM) 1991, 236, 403-410.

(11) Cyrañski, M. K.; von R. Schleyer, P.; Krygowski, T. M.; Jiao, H.; Hohlneicher, G. Tetrahedron 2003, 59, 1657-1665. cyclohexatriene structures, being in resonance, as envisaged by Kekulé $^{12}$ (first alternating $D_{3 h}$ geometries, later at the benzene $D_{6 h}$ structure). The magnetic aromaticity criterion is based on the abnormally large diamagnetic magnetizabilities, which has been rationalized as arising from the Larmor precession of electrons in (delocalized) orbits including many nuclei, known as the ring current. ${ }^{7}$

The molecular properties of molecules are well-defined and can be calculated very accurately. The magnetic properties of annulenes have been studied in detail, and on the basis of the ipsocentric formulation of induced current density, a simple orbital model could be devised that led to selection rules for ring current, which can rationalize the diatropic current found for $4 n+2 \pi$-electrons and the paratropic current for $4 n \pi$-electron annulenes. ${ }^{13,14}$ It has also been recognized that the diatropic contribution is governed by the same symmetry selection rules as those for the in-plane polarizability of a system, thus, polarizability and diatropic ring current are closely related. ${ }^{15}$ It should be noted that no relationship exists between total ring

(12) Kekulé, A. Liebigs Ann. 1872, 162, 77-124.

(13) Steiner, E.; Fowler, P. W. J. Phys. Chem. A 2001, 105, 95539562 .

(14) Steiner, E.; Fowler, P. W. Chem. Commun. 2001, 2220-2221.

(15) Soncini, A.; Fowler, P. W.; Zerbetto, F. Chem. Phys. Lett. 2005, $405,136-141$. 
SCHEME 1 Molecules under Study

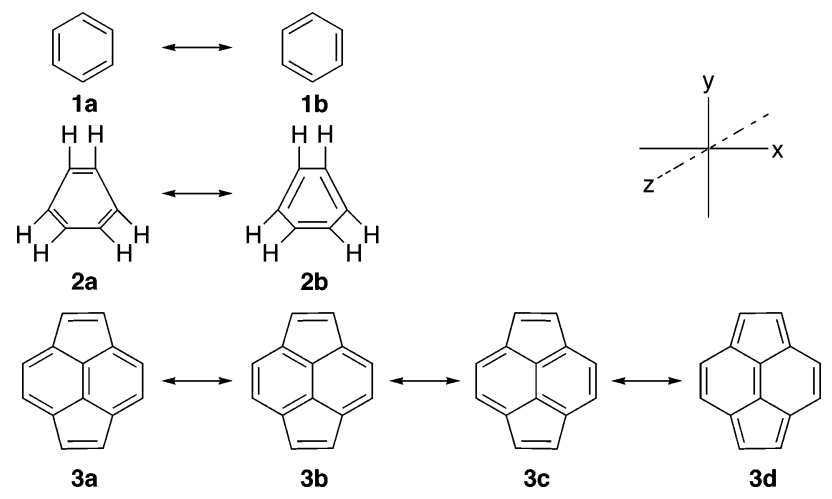

current and polarizability when the paratropic contribution to ring current becomes notable.

Different methods for the determination of resonance energy exist. The energy difference between the aromatic compound under investigation is compared to one of its nonaromatic isomers, and this difference is taken as a measure for the aromatic stabilization. The use of nonorthogonal orbitals in valence bond (VB) theory, where a bond is made by spin coupling two electrons, allows the quantum mechanical description of the different possible resonance structures and thus the calculation of the energy of one valence bond structure. The total wave function for a molecule is written as a linear combination of these structures, and hence, the energy lowering with respect to one structure due to resonance among the multiple structures can be calculated. This energy gain due to resonance is the definition of the Pauling resonance energy $\left(E_{\text {res }}\right){ }^{16}$ Although no clear relationship exists between the resonance energy and the molecular properties, empirical relationships have been found for similar molecular systems. Using Hückel theory, even an analytical relationship was derived for the series of annulenes between ring current and the Dewar resonance energy. ${ }^{17}$

The development of a response theory for valence bond wave functions ${ }^{18,19}$ enables the calculation of the polarizability and magnetizability for individual valence bond structures. This approach can be used to study the effect of resonance between structures on the molecular properties. The molecular properties can be linked to the presence or absence of induced ring currents. In this paper, valence bond response properties calculated for benzene, deformed benzene in a $D_{3 h}$ bond-alternating geometry, and a model for pyracylene (Scheme 1) are presented, to establish whether there is a direct relationship between the Pauling resonance energy and the molecular properties.

Benzene is the archetype of aromatic molecules, has a large resonance energy, ${ }^{20-22}$ and is able to sustain a diatropic ring current. ${ }^{23,24}$ Deformation of benzene to "localized" benzene leads to a loss of resonance energy, ${ }^{22}$ whereas the magnetic properties

(16) Pauling, L.; Wheland, G. W. J. Chem. Phys. 1933, 1, 362-374.

(17) Haddon, R. C. J. Am. Chem. Soc. 1979, 101, 1722-1728.

(18) Hyams, P. A.; Gerratt, J.; Cooper, D. L.; Raimondi, M. J. Chem. Phys. 1994, 100, 4408-4416.

(19) Havenith, R. W. A. Chem. Phys. Lett. 2005, 414, 1-5. 701 .

(20) Cooper, D. L.; Gerratt, J.; Raimondi, M. Nature 1986, 323, 699-

(21) Dijkstra, F.; van Lenthe, J. H.; Havenith, R. W. A.; Jenneskens, L. W. Int. J. Quantum Chem. 2003, 91, 566-574.

(22) van Lenthe, J. H.; Havenith, R. W. A.; Dijkstra, F.; Jenneskens, L. W. Chem. Phys. Lett. 2002, 361, 203-208.

(23) Fleischer, U.; Kutzelnigg, W.; Lazzeretti, P.; Mühlenkamp, V. J. Am. Chem. Soc. 1994, 116, 5298-5306. are hardly influenced. ${ }^{23,25}$ Pyracylene is interesting in the sense that the cyclopentafusion of the naphthalene core has a negligible effect on the Pauling resonance energy, ${ }^{26}$ whereas the magnetic properties change considerably as soon as the second pentagon is attached to acenaphthylene. ${ }^{27,28}$ These observations already suggest that a discrepancy exists between the magnetic and energy criteria for aromaticity.

In analogy of the calculation of pseudo $\pi$-currents for the visualization of ring current patterns for large conjugated hydrocarbons, ${ }^{29}$ the VB calculations on the pyracylene model were performed on $\mathrm{H}_{14}$ in the geometry of pyracylene, with the carbon atoms replaced by hydrogen atoms and omission of the pyracylene hydrogen atoms. Our valence bond results on the $\mathrm{H}_{14}$ model show, by comparison with previously reported VB calculations, ${ }^{26}$ that this approach is also valid for reducing the computational efforts in $\pi$-only VB calculations on (planar) conjugated hydrocarbons.

\section{Computational Details}

The geometry of benzene $\left(1, D_{6 h}\right)$ was optimized at the RHF/ $6-31 \mathrm{G}$ level of theory $\left(R_{\mathrm{C}-\mathrm{C}}=1.388 \AA\right)$. The geometry of deformed benzene $\left(2, D_{3 h}\right)$ was also optimized at the RHF/6-31G level of theory, but the $\mathrm{H}-\mathrm{C}-\mathrm{C}$ angles were fixed at $90^{\circ}$, resulting in bond length alternation in the benzene core $\left(R_{\mathrm{C}-\mathrm{C}}=1.331\right.$ and 1.524 $\AA) .{ }^{25,30}$ For the $\mathrm{H}_{14}$ geometry $\left(3, D_{2 h}\right)$, the hydrogen atoms were placed at the positions of the carbon atoms of the RHF/STO-3G optimized pyracylene geometry.

The valence bond calculations were performed with the ab initio valence bond program package TURTLE, ${ }^{31}$ which is integrated in GAMESS-UK. ${ }^{32}$ The wave functions were built from the structures depicted in Scheme 1, and the orbitals were chosen to be predominantly atomic in nature but were allowed to delocalize (cf. ref 20, delocal model). The $\sigma$-orbitals of benzene and deformed benzene were taken from preceding RHF calculations. The basis set used in the VB calculations on $\mathbf{1}$ and $\mathbf{2}$ was the 6-31G basis, and on $\mathbf{3}$, the STO-3G basis was used (the relatively small basis sets used are sufficient to draw qualitative conclusions about resonance). In all VB calculations, all orbitals were optimized for each wave function.

With the same basis set as that used in the VB calculations, the polarizability and magnetizability were also evaluated at the full $\pi$-CASSCF level of theory with DALTON,${ }^{33}$ for comparison with the properties calculated at the VB level.

2.1. Justification of the Use of the $\mathrm{H}_{14}$ Model for Pyracylene. As is shown by the work of Fowler and Steiner for, among others, pyracylene, ${ }^{29}$ the $\pi$-ring currents of polycyclic aromatic hydrocarbons can be approximated by the $\sigma$-currents obtained for model systems consisting of only hydrogen atoms at the positions of the 616.

(24) Steiner, E.; Fowler, P. W. Int. J. Quantum Chem. 1996, 60, 609-

(25) Havenith, R. W. A.; Jenneskens, L. W.; Fowler, P. W. Chem. Phys. Lett. 2003, 367, 468-474.

(26) Havenith, R. W. A.; van Lenthe, J. H.; Jenneskens, L. W. J. Org. Chem. 2005, 70, 4484-4489.

(27) Fowler, P. W.; Steiner, E.; Acocella, A.; Jenneskens, L. W.; Havenith, R. W. A. J. Chem. Soc., Perkin Trans. 2 2001, 1058-1065.

(28) Fowler, P. W.; Zanasi, R.; Cadioli, B.; Steiner, E. Chem. Phys. Lett. 1996, 251, 132-140.

(29) Fowler, P. W.; Steiner, E. Chem. Phys. Lett. 2002, 364, 259-266.

(30) Stanger, A. J. Am. Chem. Soc. 1991, 113, 8277-8280.

(31) Verbeek, J.; Langenberg, J. H.; Byrman, C. P.; Dijkstra, F.; Engelberts, J. J.; van Lenthe, J. H. TURTLE; an ab initio VB/VBSCF program; Utrecht, The Netherlands, 1988-2005.

(32) Guest, M. F.; Bush, I. J.; van Dam, H. J. J.; Sherwood, P.; Thomas, J. M. H.; van Lenthe, J. H.; Havenith, R. W. A.; Kendrick, J. Mol. Phys. 2005, 103, 719-747.

(33) DALTON, release 2.0; a molecular electronic structure program 2005. See http://www.kjemi.uio.no/software/dalton/dalton.html. 
TABLE 1. Total Energies (au), the Pauling Resonance Energy (kcal/mol), the Polarizability (au) and Magnetizability (au) for Benzene $(1 \mathrm{a}+1 \mathrm{~b})$, and Its Kekulé Resonance Structures (1a/1b)

\begin{tabular}{lcrrrrrr}
\hline & & & \multicolumn{2}{c}{ polarizability } & & \multicolumn{2}{c}{ magnetizability } \\
\cline { 5 - 6 } structure & energy & \multicolumn{1}{c}{$E_{\text {res }}$} & $\alpha_{x x}, \alpha_{y y}$ & $\alpha_{z z}$ & & $\chi_{x x}, \chi_{y y}$ & $\chi_{z z}$ \\
\hline 1a + 1b & -230.6927260 & 20.00 & 61.15 & 18.14 & & -40.63 & -52.83 \\
1a, 1b & -230.6776060 & 0.00 & 62.91 & 18.20 & & -40.57 & -53.00 \\
CASSCF & -230.7001172 & & 62.53 & 18.14 & & -40.64 & -53.10 \\
\hline
\end{tabular}

carbon atoms, with considerable reduction in computer time. The VB property calculations on pyracylene itself are still computationally too demanding, thus the VB properties were calculated using the same $\mathrm{H}_{14}$ model as had been used for the calculation of the pseudo $\pi$-ring currents. The weights of the structures calculated for this model are 0.646 for $\mathbf{3 a}, 0.173$ for $\mathbf{3 b} / \mathbf{3 c}$, and 0.008 for $\mathbf{3 d}$ (Scheme 1), which is remarkably close to those obtained for pyracylene itself (3a: $0.642, \mathbf{3 b} / \mathbf{3 c}: \quad 0.175$, and $\mathbf{3 d}: 0.008){ }^{26}$ The relative energies of the structures obtained for the $\mathrm{H}_{14}$ model (3a: $0.0 \mathrm{kcal} / \mathrm{mol}, \mathbf{3 b} / \mathbf{3 c}$ : $62.8 \mathrm{kcal} / \mathrm{mol}$, and $\mathbf{3 d}: 165.0 \mathrm{kcal} / \mathrm{mol}$ ) are also in agreement with those found in the $\pi$-VB calculation on 3 (3a: $0.0 \mathrm{kcal} / \mathrm{mol}, 3 \mathrm{~b} / 3 \mathrm{c}$ : $52.6 \mathrm{kcal} / \mathrm{mol}$, and 3d: $167.6 \mathrm{kcal} / \mathrm{mol}$ ). Coincidentally, the Pauling resonance energy of $15.28 \mathrm{kcal} / \mathrm{mol}$ found for $\mathrm{H}_{14}$ is similar to the value of $10.6 \mathrm{kcal} / \mathrm{mol}$ calculated for pyracylene.

All these resemblances in VB results for the $\mathrm{H}_{14}$ model and pyracylene suggest that this model can also be used for the study of the properties of the individual resonance structures in relation with resonance, while saving computation time.

\section{Results and Discussion}

3.1. Benzene $\left(\boldsymbol{D}_{\boldsymbol{6} h}\right)$. The valence bond results obtained for benzene are in agreement with previously reported results. ${ }^{20-22}$ It has a substantial Pauling resonance energy of $20.00 \mathrm{kcal} /$ mol (Table 1), and both Kekulé structures are equally important in the VB wave function. The one-structure description is, obviously, not stabilized by resonance and has $9.49 \mathrm{kcal} / \mathrm{mol}$ higher energy.

The molecular properties of benzene calculated at the twostructure VB level are in good agreement with those obtained at the CASSCF (Table 1) and spin-coupled levels of theory. ${ }^{19,34}$ The one-structure description gives virtually identical properties to those of the two-structure description. The small deviations are due to small changes in the orbitals. The CI space does not contribute. This may be seen by a symmetry consideration. The CI space consists of two states, viz. $\Psi_{0}=N\left(\Phi_{\mathbf{1 a}}+\Phi_{\mathbf{1 b}}\right)\left(A_{1 g}\right.$ symmetry) and $\Psi_{1}=N\left(\Phi_{1 \mathbf{a}}-\Phi_{1 \mathbf{b}}\right)\left(B_{2 u}\right.$ symmetry $)$. The electric field response is governed by the dipole moment operators $(\hat{\mathrm{O}}=\hat{\mathrm{x}}, \hat{\mathrm{y}}, \hat{\mathrm{z}})$, which possess $E_{1 u}$ and $A_{2 u}$ symmetry. The CI response is determined by the matrix element $\left\langle\Psi_{0}|\hat{O}| \Psi_{1}\right\rangle$, which vanishes by symmetry. Equally, for the magnetizability, the CI response also vanishes by symmetry, as it is determined by the angular momentum operators, which have $A_{2 g}$ and $E_{1 g}$ symmetry. Thus, the total molecular properties for the two-structure description are the weighted averages of the properties of the individual resonance structures, ${ }^{19}$ which are identical by symmetry. Hence, the response properties of benzene are equal to those of one of its two Kekule resonance structures.

In a description in which the p-orbitals are not allowed to delocalize but are constrained to remain completely atomic (strictly atomic model), the in-plane orbital response is consider-

(34) Hyams, P. A. Ph.D. Thesis, University of Bristol, U.K., 1990.
SCHEME 2 Sets of Ionic Structures ${ }^{a}$
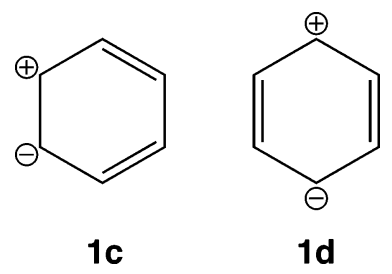

${ }^{a}$ Only the symmetrically unique structures are shown. Set $\mathbf{1 c}$ consists of a total of 12 structures, and set 1d consists of a total of 6 structures.

TABLE 2. Polarizability Calculated for Benzene Using Strictly Atomic $\mathbf{p}_{\pi}$-Orbitals

\begin{tabular}{lccc}
\hline \multicolumn{1}{c}{ structure } & energy & \multicolumn{2}{c}{ polarizability } \\
\cline { 2 - 4 } & $\alpha_{x x}, \alpha_{y y}$ & $\alpha_{z z}$ \\
\hline $\mathbf{1 a}+\mathbf{1 b}$ & -230.5406776 & 41.40 & 19.29 \\
$\mathbf{1 a}+\mathbf{1 b}+\mathbf{1 c}$ & -230.6364127 & 49.97 & 18.49 \\
$\mathbf{1 a}+\mathbf{1 b}+\mathbf{1 d}$ & -230.5708882 & 45.23 & 19.30 \\
$\mathbf{1 a}+\mathbf{1 b}+\mathbf{1 c}+\mathbf{1 d}$ & -230.6527610 & 54.28 & 18.43 \\
\hline
\end{tabular}

TABLE 3. Total Energies (au), the Pauling Resonance Energy (kcal/mol), the Polarizability (au) and Magnetizability (au) for Deformed Benzene $(2 a+2 b)$, and Its Kekulé Resonance Structures $(2 \mathbf{a} / 2 \mathbf{b})$

\begin{tabular}{lccccccc}
\hline & & & \multicolumn{2}{c}{ polarizability } & & \multicolumn{2}{c}{ magnetizability } \\
structure & energy & $E_{\text {res }}$ & $\alpha_{x x}, \alpha_{y y}$ & $\alpha_{z z}$ & & $\chi_{x x}, \chi_{y y}$ & $\chi_{z z}$ \\
\hline $\mathbf{2 a}+\mathbf{2 b}$ & -230.4658096 & 3.50 & 63.20 & 18.34 & & -41.88 & -51.56 \\
$\mathbf{2 a}$ & -230.4611314 & 0.00 & 63.58 & 18.34 & -41.93 & -51.27 \\
2b & -230.4278598 & 0.00 & 65.98 & 18.57 & & -41.42 & -53.10 \\
CASSCF & -230.4703118 & & 63.86 & 18.35 & & -41.84 & -51.84 \\
\hline
\end{tabular}

ably smaller. ${ }^{19}$ The delocal model includes ionic structures, due to the optimization of the p-orbitals, which are not confined to one atomic center. To obtain proper response properties using the strictly atomic model, it is necessary to explicitly include ionic structures in the wave function ${ }^{35}$ (mimicking the delocal orbital optimization). The ionic states added to the wave function span the symmetries of the property operators, thus their CI response contribution is nonnegligible. To elucidate which ionic structures are important, the polarizability of benzene was calculated with wave functions consisting of the two Kekule resonance $(\mathbf{1 a}+\mathbf{1 b})$ structures and the sets of ionic structures depicted in Scheme 2.

The results in Table 2 show that when only the two Kekulé resonance structures are used indeed the in-plane polarizability significantly decreases in comparison with the delocal VB or CASSCF results. Neither the structure 1c nor $\mathbf{1 d}$ alone gives rise to a proper in-plane polarizability, but when both sets of structures are included in the wave function, the polarizability approaches the delocal and CASSCF results.

3.2. Deformed Benzene $\left(D_{3 h}\right)$. Optimization of benzene with a constrained $\mathrm{H}-\mathrm{C}-\mathrm{C}$ angle of $90^{\circ}$ leads to a $D_{3 h}$ structure with alternating $\mathrm{C}-\mathrm{C}$ bond lengths of 1.331 and $1.524 \AA . .^{25,30}$ The weights of the resonance structures $\mathbf{2} \mathbf{a}$ and $\mathbf{2} \mathbf{b}$ in the VB wave function are 0.866 and 0.134 , respectively. The structure 2a, which has the double bonds along the shorter $\mathrm{C}-\mathrm{C}$ bond, is the most important structure in the VB wave function and has the lowest energy (Table 3). The Pauling resonance energy dramatically decreases to $3.50 \mathrm{kcal} / \mathrm{mol}$ upon deformation,

(35) Shurki, A.; Hiberty, P. C.; Dijkstra, F.; Shaik, S. J. Phys. Org. Chem. 2003, 16, 731-745. 
TABLE 4. Total Energies (au), the Pauling Resonance Energy (kcal/mol), the Polarizability (au) and Magnetizability (au) for the $\mathrm{H}_{14}-\mathrm{Model}$ for Pyracylene $(3 \mathbf{a}+\mathbf{3 b}+\mathbf{3} \mathbf{c}+\mathbf{3 d})$, and Its Kekulé Resonance Structures $(\mathbf{3 a} / \mathbf{3 b} / \mathbf{3 c} / \mathbf{3 d})$

\begin{tabular}{|c|c|c|c|c|c|c|c|c|}
\hline \multirow[b]{2}{*}{ structure } & \multirow[b]{2}{*}{ energy } & \multirow[b]{2}{*}{$E_{\mathrm{res}}$} & \multicolumn{3}{|c|}{ polarizability } & \multicolumn{3}{|c|}{ magnetizability } \\
\hline & & & $\alpha_{x x}$ & $\alpha_{y y}$ & $\alpha_{z z}$ & $\chi_{x x}$ & $\chi_{y y}$ & $\chi_{z z}$ \\
\hline $3 a+3 b+3 c+3 d$ & -7.0997395 & 15.28 & 43.69 & 31.55 & 0.00 & -43.68 & -30.94 & -53.04 \\
\hline $3 a$ & -7.0785043 & 0.00 & 46.02 & 32.69 & 0.00 & -43.64 & -30.98 & -52.99 \\
\hline $3 b / 3 c$ & -7.0406052 & 0.00 & 46.99 & 35.86 & 0.00 & -43.76 & -30.90 & -53.29 \\
\hline 3d & -6.9017472 & 0.00 & 28.78 & 61.90 & 0.00 & -43.42 & -31.42 & -45.95 \\
\hline CASSCF & -7.1238830 & & 47.58 & 36.72 & 0.00 & -43.69 & -30.93 & -52.50 \\
\hline
\end{tabular}

compared to the benzene value of $20.00 \mathrm{kcal} / \mathrm{mol}$, suggesting a substantial reduction of aromatic character. However, as shown previously, the ring current survives bond alternation and remains nearly as strong as in benzene. . $3,25,36,37^{2}$

Again, the VB response properties are in agreement with CASSCF results and in line with previous findings that deforming benzene to a bond alternating structure does not influence the magnetizability substantially. ${ }^{23}$ No appreciable change in both molecular properties at the VB level of theory is found when benzene is deformed. The polarizability and magnetizability of this deformed benzene are similar to those for the most important structure $\mathbf{2} \mathbf{a}$, and the properties of both Kekulé resonance structures are similar in magnitude. Thus, although the Pauling resonance energy decreases considerably and structure $\mathbf{2} \mathbf{a}$ becomes more important in the wave function, the total response properties are much less influenced.

3.3. $\mathrm{H}_{14}$ Model for Pyracylene. A reasonable agreement is found between the total VB properties and the CASSCF properties (Table 4). Note that the $\alpha_{z z}$ value of 0.00 au is caused by the small STO-3G basis, which lacks p-functions, which are necessary for a contribution to the polarizability in this direction. The three resonance structures $\mathbf{3 a}-\mathbf{c}$ all have identical response properties. The polarizability of structure 3d (Scheme 1) differs considerably from those found for the other structures. However, the different properties of $\mathbf{3 d}$ have only a marginal effect on the total properties of the molecule because of its small weight in the total wave function. The most important structures $\mathbf{3 a}$, $\mathbf{3 b}$, and $\mathbf{3 c}$ already possess the response properties of the whole molecule, and resonance between them does not play an important role in the response properties.

Despite the small weight of structure $\mathbf{3 d}$, it is still interesting to see why its polarizability differs from the others, considering the fact that for the other different spin-coupling schemes similar properties are obtained. As shown by perturbation theory, lowlying excited states contribute more to the total property than higher-lying excited states. Assuming that ionic states similar to those for benzene (1) are needed in the wave function of $\mathbf{3}$ to obtain the correct properties, then inspection of the nature of these ionic states already explains why the response of structure 3d differs from the others. In Scheme 3, these ionic structures are drawn for structure $\mathbf{3 a}, \mathbf{3 b}$, and $\mathbf{3 c}$. For the resonance structures $\mathbf{3 a}-\mathbf{c}$, closed-shell, ionic structures can be drawn, which are similar to each other and to those of benzene. However, for resonance structure 3d, because of the difference in spin coupling, these states have biradical character and thus a higher energy. Consequently, their contribution to the polarizability will be smaller, and the total polarizability of structure $\mathbf{3 d}$ will differ significantly from those of $\mathbf{3 a}-\mathbf{c}$.

(36) Fowler, P. W.; Havenith, R. W. A.; Jenneskens, L. W.; Soncini, A.; Steiner, E. Chem. Commun. 2001, 2386-2387.

(37) Soncini, A.; Havenith, R. W. A.; Fowler, P. W.; Jenneskens, L. W.; Steiner, E. J. Org. Chem. 2002, 67, 4753-4758.
SCHEME 3 Ionic States for the Resonance Structures 3a,b,d that are Similar to Those of Benzene and Dominate Its Polarizability in the $x$ - and $y$-Direction

$3 a$<smiles>C1=c2cc[14cH]c3ccc4ccc1c2c4-3</smiles><smiles>C1=C[C@@H]2C=Cc3c1ccc1c3C2C=C1</smiles>

$3 \mathbf{b}$<smiles></smiles><smiles></smiles>

$3 d$
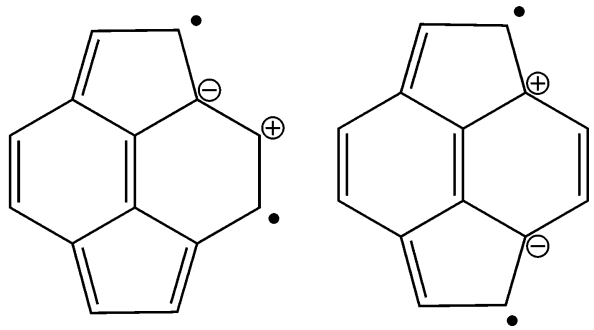

\section{Implications for Ring Current and for Interpretation of Valence Bond Structures}

The existence of ring current is reflected in the magnetizability of a compound. Therefore, predictions regarding the existence of induced ring currents in the individual Kekulé resonance structures can be drawn.

Both the polarizability and magnetizability of the individual structures are close to those obtained for the "all-structure" wave function, which suggests that the ring current patterns, if calculated for one structure only, would be identical to those obtained at the RHF or CASSCF levels. Thus, a one-structure description of benzene would be aromatic based on the magnetic criterion for aromaticity. These results indicate that, although an empirical relationship exists between the nucleus-independent chemical shift (NICS) and the aromatic stabilization energy, ${ }^{9}$ no direct relationship exists between the Pauling resonance energy and induced ring current. This implies that criteria based on response properties and the Pauling resonance energy can lead to different conclusions regarding aromaticity (see also ref 3 ). Both definitions can be fulfilled for a compound at the same time, but that is not necessarily the case.

The aromaticity based on the ring-current criterion of onestructure benzene suggests that the spin-coupled bonds in a 
valence bond structure do not behave like localized bonds in this respect, as the properties evaluated for one-structure benzene are similar to those of benzene and do not match with values obtained using bond increments. ${ }^{23}$ Thus, the intuitive correspondence between a valence bond and a Lewis structure, with localized bonds, does not exist, and the spin-coupled bonds are not independent, localized bonds. Separation of the two structures is difficult, as indicated by their overlap of $67 \%$ (spin overlap already $25 \%$ ), and the one-structure wave function has a remarkably high overlap of $93 \%$ with the Hartree-Fock wave function. This high overlap suggests that the properties evaluated using the one-structure VB wave function are very similar to those evaluated using the Hartree-Fock wave function. However, localization of the geometry to a $D_{3 h}$ benzene occurs upon optimization of the geometry using one structure, ${ }^{21,22}$ indicating that spin coupling still increases the bond strength in one direction, in line with geometrical criteria for aromaticity.

\section{Conclusions}

The electric and magnetic response properties for benzene (1), deformed benzene (2), and model $\mathrm{H}_{14}$ pyracylene (3) and their individual Kekulé resonance structures have been evaluated using valence bond theory. The results show that the polarizability and magnetizability for the molecules considered here are already obtained from one structure, without any resonance. There is no direct relationship between the Pauling resonance energy and response properties. Resonance between the structures does not influence the response properties, and these properties are weighted averages of the response properties of the individual resonance structures.

Acknowledgment. Dr. J. H. van Lenthe (Utrecht University) is gratefully acknowledged for helpful discussions, and Dr. D. L. Cooper (University of Liverpool) is gratefully acknowledged for providing a copy of P. A. Hyams' thesis. I gratefully acknowledge partial financial support from NWO/NCF for the use of supercomputer time on TERAS/ASTER, SARA (The Netherlands, project number SG-032), and financial support from The Netherlands Organization for Scientific Research (NWO), grant 700.53.401.

JO060249K 